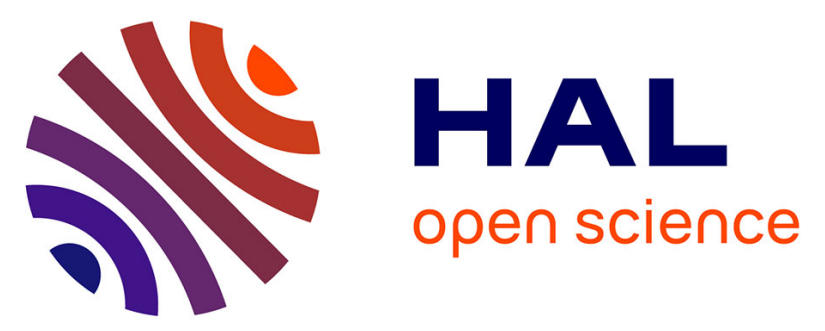

\title{
Paradoxical High-Level Spiramycin Resistance and Erythromycin Susceptibility Due to 23S rRNA Mutation in Streptococcus constellatus
}

Agathe Capitaine, Paul-Louis Woerther, Michel Auzou, Elisabeth Chachaty, François Guerin, Jean-Christophe Giard, Vincent Cattoir, Christophe Isnard

\section{To cite this version:}

Agathe Capitaine, Paul-Louis Woerther, Michel Auzou, Elisabeth Chachaty, François Guerin, et al.. Paradoxical High-Level Spiramycin Resistance and Erythromycin Susceptibility Due to 23S rRNA Mutation in Streptococcus constellatus. Microbial Drug Resistance, 2020, 26 (7), pp.727-731. 10.1089/mdr.2019.0288. hal-02569879

\section{HAL Id: hal-02569879}

https://hal-normandie-univ.archives-ouvertes.fr/hal-02569879

Submitted on 9 Jun 2020

HAL is a multi-disciplinary open access archive for the deposit and dissemination of scientific research documents, whether they are published or not. The documents may come from teaching and research institutions in France or abroad, or from public or private research centers.
L'archive ouverte pluridisciplinaire HAL, est destinée au dépôt et à la diffusion de documents scientifiques de niveau recherche, publiés ou non, émanant des établissements d'enseignement et de recherche français ou étrangers, des laboratoires publics ou privés. 
1 Paradoxical high-level spiramycin resistance and erythromycin

2 susceptibility due to 235 rRNA mutation in Streptococcus

3 constellatus

4

5 Agathe Capitaine ${ }^{1}$, Paul-Louis Woerther $^{2}$, Michel Auzou $^{1}$, Elisabeth Chachaty ${ }^{3}$, 6 François Guérin ${ }^{1,4}$, Jean-Christophe Giard ${ }^{5}$, Vincent Cattoir ${ }^{6,7}$ and Christophe 7 Isnard $^{1,4, *}$

Running title: Paradoxical MLS resistance due to 235 rRNA mutation in Streptococcus constellatus

Normandie Univ, UNICAEN, CHU de CAEN Normandie, Service de Microbiologie, 14000 Caen, France ${ }^{1}$; Unité de Bactériologie-Hygiène, Département de Microbiologie, Assistance Publique - Hôpitaux de Paris, Hôpital Henri Mondor, Créteil, France ${ }^{2}$; Service de Microbiologie, Institut Gustave Roussy APHP, Villejuif, France ${ }^{3}$; Normandie Univ, UNICAEN/UNIROUEN, EA2656 GRAM2.0, 14000 Caen, France 4; Normandie Univ, UNICAEN, EA4655 U2RM, 14000 Caen, France ; Centre Hospitalier Universitaire de Rennes, Site Pontchaillou, 35000 Rennes, France ${ }^{6}$; CNR de la Résistance aux Antibiotiques, Laboratoire Associé « Entérocoques et résistances particulières chez les bactéries à Gram positif », 35000 Rennes, France?.

*Correspondance : Mailing address: Dr. Christophe ISNARD, Service de Microbiologie, CHU de CAEN, 14033 Caen Cedex, France.

E-mail: isnard-c@chu-caen.fr 
Keywords: Streptococcus constellatus; Streptococcus milleri group; MLS resistance; 23S rRNA mutation; A2062C; ARMS qPCR.

Word counts: Abstract: 253; Text: 1942; Tables: 2; Figures: 1; References: 19

\section{Abstract}

Objectives: The aim of the study was to characterize phenotypically and genotypically an uncommon mechanism of resistance to macrolides, lincosamides and streptogramins (MLS) in a Streptococcus milleri group clinical isolate.

Methods: The isolate UCN96 was recovered from an osteoradionecrosis wound, and was identified using the MALDI-TOF mass spectrometry and the partial sequencing of the sodA gene. Antimicrobial susceptibility testing (AST) were carried out by the disc diffusion method and MICs were determined by the broth microdilution technique. PCR screening was performed for MLS resistance genes described in Gram-positive bacteria. Specific mutations in the ribosomal proteins L3-, L4- and L22-encoding genes were also screened as well as those in domain $V$ of the $23 \mathrm{~S}$ rRNA gene $(r r l)$. The number of mutated copies of the $r r l$ gene was determined using ARMS (amplification-refractory mutation system) qPCR analysis.

Results: The clinical isolate UCN96 was unambiguously identified as Streptococcus constellatus. It was susceptible to all ML antibiotics except spiramycin (MIC >256 $\mathrm{mg} / \mathrm{L}$ ) while it was also resistant to streptogramins. Screening for all acquired resistance genes was negative while no mutation was found in genes coding for $L 3$, L4 and L22 ribosomal proteins. Interestingly, a single mutation, A2062C (according to Escherichia coli numbering), was detected in the domain $\mathrm{V}$ of $23 \mathrm{~S}$ rRNA.

Conclusion: Mutations at the position 2062 of 23S rRNA have been detected once in S. pneumoniae, and not yet in other Streptococcus spp. This mechanism is very 
51 likely uncommon in Gram-positive bacteria since different copies of 23S rRNA 52 operons should be mutated for development of such a resistance pattern. 


\section{Introduction}

Streptococcus constellatus belongs to the Streptococcus milleri group (SMG) besides two others species, Streptococcus anginosus and Streptococcus intermedius. SMG is part of the human microbiota and can be in the oral cavity as well as abdominal, urogenital and upper respiratory tracts ${ }^{1}$. Streptococci from the SMG are responsible for various pyogenic infections including cardiac, skin, abdominal and central nervous system infections ${ }^{1}$. These organisms are usually susceptible to many antimicrobial agents including those commonly used for infections caused by streptococci such as $\beta$-lactams, macrolides-lincosamides-streptogramins (MLS) and glycopeptides.

Whereas there is no resistance to $\beta$-lactams and glycopeptides described in SMG, acquired MLS resistance has been reported, with approximately $17 \%$ of clinical isolates resistant to erythromycin and clindamycin ${ }^{2}$. In streptococci, MLS resistance is mediated by two major mechanisms which are target site modification and active drug efflux ${ }^{3}$. Ribosomal alteration is caused by methylation of a specific adenine residue (the so-called A2058) of target site in the 23S rRNA and is mediated by an adenine-N6-methyltransferase encoded by a gene belonging to the erm class (erythromycin ribosome methylase). This methylation of the A2058 residue induces a conformational change in the $50 \mathrm{~S}$ ribosomal subunit that alter the binding of antibiotics to the ribosome and causes the so-called $\mathrm{MLS}_{\mathrm{B}}$ phenotype. The second mechanism consisted of an active efflux of the antibiotics encoded by the mef gene. This active efflux is responsible for unique resistance to 14- and 15-membered macrolides and confers the so-called M phenotype. Other mechanisms of MLS resistance have been rarely described in Gram-positive bacteria, such as other mutations in 23S rRNA or in L3, L4, L22 ribosomal proteins and to our knowledge, have not been yet described in the SMG ${ }^{4-8}$. 
78 The aim of this study was to decipher the molecular aspect of an atypical 79 resistance phenotype in a clinical isolate of S. constellatus (UCN96).

80

81 (Part of this work was presented in the $14^{\text {th }}$ European Congress of Clinical 82 Microbiology and Infectious Diseases, EV0187, Copenhagen, 2015) 


\section{Materials \& Methods}

\section{Bacterial strains used in the study}

The UCN96 isolate was recovered in June 2014 from a osteoradionecrosis radionecrosis wound in a patient hospitalized at the Gustave Roussy Institute (Villejuif, France) for a tongue cancer medical history. The same bacterial strain was recovered from the patient in a chin outpouring in May 2014 but the antimicrobial susceptibility testing realized then, showed that the $S$. constellatus isolated was fully susceptible to all MLS antibiotics. In front of this statement, the patient has received pristinamycin at $1 \mathrm{~g}$ twice in a day for 10 days since he was intolerant to $\beta$-lactams. The isolate UCN96 was identified by MALDI-TOF mass spectrometry (Microflex; Brucker Daltonics; Germany) and partial sequencing of the sodA gene as previously described ${ }^{9}$. Staphylococcus aureus ATCC 29213 and Streptococcus pneumoniae ATCC 49619 were used as antimicrobial susceptibility testing (AST) controls while an MLS-susceptible S. constellatus from our collection (S. constellatus 13422) was also tested as wild-type control for comparative purposes.

\section{Antimicrobial susceptibility testing}

Antimicrobial susceptibility testing was carried out by the disc diffusion method using Mueller-Hinton agar plates supplemented with lysed horse blood (5\%) and $\beta-N A D$ (20 mg/L) (bioMérieux; France) according to the Antibiogram Committee of the French Society for Microbiology recommendations (CA-SFM/EUCAST; www.sfmmicrobiologie.org). MICs of erythromycin, azithromycin, spiramycin, josamycin, telithromycin, lincomycin, clindamycin, pristinamycin, quinupristin-dalfopristin, quinupristin, dalfopristin and chloramphenicol were determined by the broth microdilution technique according to EUCAST guidelines (http://eucast.org/). 
110 DNA extraction was performed using the automated easyMAG ${ }^{\circledR}$ extractor 111 (bioMérieux, France) according to the protocol suggested by the manufacturer.

112 Extracted bacterial DNA was eluted with $25 \mu$ l elution buffer and stored at $-20^{\circ} \mathrm{C}$. 113 PCR experiments for the detection of acquired genes putatively involved in MLS 114 resistance [e.g. $\operatorname{erm}(\mathrm{A}), \operatorname{erm}(\mathrm{B}), \operatorname{erm}(\mathrm{C}), \operatorname{erm}(\mathrm{F}), \operatorname{erm}(\mathrm{TR}), \operatorname{erm}(\mathrm{T}), \operatorname{erm}(\mathrm{X}), \operatorname{msr}(\mathrm{A})$ and $\operatorname{mef}(\mathrm{A})]$ as well as uncommon acquired genes $[\ln u(\mathrm{~A}), \ln u(\mathrm{~B}), \ln u(\mathrm{C}), \ln u(\mathrm{E})$, Isa(A), Isa(B), Isa(C), Isa(E), vat(A), vat(B), vat(C), vat(D), vat(E), vga(A), vga(B), $v g a(\mathrm{C}), \operatorname{vga}(\mathrm{D}), \operatorname{vga}(\mathrm{E}), \operatorname{vg} b(\mathrm{~A}), \operatorname{vg}(\mathrm{B})]$ were carried out using an in-house method. The screening of point mutations was also performed by PCR-sequencing using specific primers (Sigma-Aldrich, France) (Table 1) allowing amplification of the L3, L4 and L22 ribosomal protein encoding genes (rp/C, rp/D and $r p / V$ genes respectively) and in the 23S rRNA ( $r r l)$ gene. Amplification refractory mutation system (ARMS) qPCR was used to distinguished if the mutation of $r r l$ gene affected all the alleles harbored by the bacterial strain (e.g. 4 alleles in S. constellatus and all SMG) as previously described in eucaryotes ${ }^{10,11}$. Primers used for qPCR ARMS experiment are detailed in Table 1. Briefly, two sets of primers were design. Oligonucleotide sequences of the forward primers were strictly complementary to the $r r l$ region where the mutation was found. For one of the two forward primers, the nucleotide localized at the 3' end corresponded to the nucleotide found in the mutant strain sequence, whereas for the other, it corresponded to the nucleotide found in the wild type. The reverse primer was the same for the 2 experiments. ARMS qPCR experiments were made by real-time PCR using the SsoAdvanced universal SYBR green supermix kit and a CFX Connect real-time PCR detection system (Bio-Rad, France) according to manufacturer's recommendations. To determine if the mutation appeared in several 
gene loci, a comparison of cycle threshold between the two sets of primers was analyzed for the mutated strain UCN96. A susceptible strain of our collection, $S$. constellatus 13422, was used as comparator. In case of higher initial amount of mutated DNA matrix, a lower CT value should be obtained. Then if a difference in CT values was observed between the 2 sets of primers, we should conclude that more than one allele was mutated. Conversely, the same result was expected in favor of the "wild-type" set of primers when using the S. constellatus 13244 DNA as template.

\section{Multiple alignment, phylogenetic and gene analysis.}

Genome of the annotated strain S. constellatus 1050 available at the National Center for Biotechnology Information (http://www.ncbi.nlm.nih.gov/genome/) was used for comparative sequences analysis. 23S rRNA gene partial sequence was then compared to deposited sequences available from the BIBI site database (https://umr5558-bibiserv.univ-lyon1.fr/lebibi/lebibi.cgi). Sequence comparison by multiple alignment and phylogenetic analysis were performed using BioEdit Sequence Alignment Editor Software (http://www.mbio.ncsu.edu/bioedit/bioedit.html) and the neighbour-joining algorithm with the ClustalOmega software (http://www.ebi.ac.uk/Tools/msa/clustalo/). 


\section{Results}

\section{Bacterial Identification}

The identification of strain UCN96 was carry out twice using the MALDI-TOF mass spectrometry technology and was unambiguously confirmed as S. constellatus by the partial sodA gene sequencing.

\section{In vitro antimicrobial susceptibility testing}

By the disc diffusion method, the strain UCN96 was susceptible to almost all tested antimicrobials: penicillin G, ampicillin, kanamycin, tobramycin, gentamicin, vancomycin, teicoplanin, levofloxacin, rifampicin, tetracycline, chloramphenicol and cotrimoxazole but in contrast, it exhibited an uncommon MLS resistance. After MIC determination, the strain appeared harbouring an a priori paradoxical $\mathrm{MS}_{\mathrm{AB}}$ resistance phenotype. Indeed, the strain was susceptible to all 14- and 15-membered macrolides (e.g. erythromycin, telithromycin, and azithromycin, with MICs of 0.03 , $<0.008$ and $0.12 \mathrm{mg} / \mathrm{L}$, respectively), lincosamides (e.g. lincomycin and clindamycin, with MICs of 1 and $0.06 \mathrm{mg} / \mathrm{L}$, respectively) and chloramphenicol with a MIC of 8 $\mathrm{mg} / \mathrm{L}$. On the other hand, it was highly-resistant to spiramycin or josamycin (16membered macrolides) with MICs of $>256$ and $32 \mathrm{mg} / \mathrm{L}$, respectively and streptogramins (MIC >64 mg/L; $8 \mathrm{mg} / \mathrm{L}, 4 \mathrm{mg} / \mathrm{L}$ and $8 \mathrm{mg} / \mathrm{L}$ for dalfopristin, quinupristin, quinupristin-dalfopristin and pristinamycin, respectively.) (Table 2). As compared to S. constellatus 13422 susceptible strain, UCN96 exhibited MICs of spiramycin, josamycin, dalfopristin, quinupristin, quinupristin-dalpristin and pristinamycin 4, 512, 32, 8, 8 and 64-fold higher, respectively (Table 2).

\section{Molecular characterization of the uncommon resistance phenotype}


178 PCR experiments for the detection of acquired genes putatively involved in MLS 179 resistance as well as uncommon acquired resistance patterns were all negative and 180 no mutation was found in L3, L4 and L22 ribosomal protein-encoding genes. 181 Interestingly, the strain displayed a point mutation in the $r r l$ gene at an unexpected 182 position. Indeed, a nucleotide transition was found at position 2062 (E. coli 183 numbering) (A2062C) as shown in figure 1A. Considering that $S$. constellatus harbored four copies of the ribosomal operon we assessed if the mutation affected one or several $r r$ operons. The ARMS qPCR technique was used and confirmed the presence of the A2062C mutation in more than a single copy of the 23S rRNA gene in the strain UCN96. When DNA extracted from the resistant strain UCN96 was used as template, cycle threshold (CT) obtained with the set of mismatches primers appeared sooner (e.g. fourteen times), than the CT obtained with the set of primers designed with the wild type sequence of the $r r$ operon (Figure 1B right). Inversely, when the DNA extracted from the susceptible strain was used as template, only a six CT difference was observed between the two sets of primers in favor of the unmismatches primers (Figure 1B left). Such results, allow us to strongly suggest that the point mutation $\mathrm{A} 2062 \mathrm{C}$ was present in more than one $23 \mathrm{~S}$ rRNA operon 195 copy. 


\section{Discussion}

198 S. constellatus can be found as a part of the oral cavity and upper respiratory tract flora but can be also responsible for various pyogenic infections where a therapeutic management is required ${ }^{1}$. $\beta$-lactams are the first-line molecules recommended for the treatment of streptococcal infections but in patients intolerant to that class of antibiotics, MLS antimicrobials constitute an alternative therapy.

The target site for MLS molecules is the 50 S ribosomal subunit ${ }^{12}$ and many cases of MLS resistance could be linked with an alteration by methylation of 235 rRNA at positions 2058 and/or $2059^{3,6}$. In bacteria harboring several rRNA operon copies (such as Staphylococcus spp., Streptococcus spp. or Enterococcus spp.) the most prevalent mechanism for MLS resistance is an acquired mechanism consisting in expression of erm gene encoding methyltransferase or active efflux of the antibiotics encoded by the mef gene ${ }^{3,7,13,14}$. Point mutations in $23 S$ rRNA have been reported and appeared at specific positions of the domain $\mathrm{V}$ especially in pathogens harboring only 1 or 2 rRNA operon copies, such as mycoplasma with a prevalence in Mycoplasma genitalium estimated around $50 \%{ }^{15}$. These mutations, regarding the species and nucleotide substitution, conferred different phenotypes of resistance. To our best knowledge, MLS resistance linked to these types of mutations seemed to be less prevalent in Gram-positive bacteria likely because they harbored a higher number of rRNA operons (4 rrn operons in streptococci or enterococci for example) and appeared generally at position 2058, 2059 or 2611, according to the species 218 involved ${ }^{7}$. Interestingly, in our S. constellatus clinical isolate, the mutation found (A2062C) seemed to be very uncommon since it has been reported only once in a spiramycin- and streptogramin-resistant strain of Streptococcus pneumoniae and in a chloramphenicol-resistant Halobacterium halobium ${ }^{16,17,18}$. Surprisingly, whereas $H$. 
halobium harbored a single copy of rRNA operon, S. pneumoniae harbored 4 copies (like S. constallatus) and the mutation was found in the four copies of this operon $16,18,19$. Our data were in accordance with these observations since several copies of rrl operon seemed altered in S. constellatus UCN96. Then, clinical isolates of viridans streptococci that harbored this unusual phenotype of resistance (susceptibility to 14-15 membered macrolides and lincosamides, resistance to 16membered macrolides and streptogramins) has not been reported yet. Moreover, as previously described by different studies concerning the crystal structure of the interaction area of MLS antibiotics with $50 \mathrm{~S}$ SU, amino acids modification in position 2062 (transition to a nonpolar to a polar amino acid) possibility changed hydrogen links between MLS antibiotics and their target in 50S SU of Staphylococcus aureus ribosome. As a consequence, resistance to different MLS antibiotics should be explained by this conformational variation ${ }^{20}$.

As a conclusion, we have identified and characterized a point mutation (A2062C) found in more than one locus of the domain $V$ of $23 \mathrm{~S}$ rRNA that confers spiramycin and streptogramins resistance in S. constellatus isolated from a patient suffering of radionecrosis wound and treated with pristinamycin. Since S. constellatus is part of the oral cavity and upper respiratory tract flora and that irradiation is largely use in head and neck cancers, these data suggest that in clinical strains of SMG in patients that previously received MLS therapy, it would be wise to follow the susceptibility of the strain concerning all the MLS class of drugs (e.g. 14-, 15- and 16- membered macrolide, ketolide and also to lincosamide and streptogramins). 
245 Author Disclosure Statement

246 The authors declare that they have no conflicts of interest.

247

$248 \quad$ Funding

249 This work was supported by a grant from the Ministère de l'Enseignement Supérieur 250 et de la Recherche to EA4655, Université de Caen Normandie, France.

251 


\section{References}

1. Gossling, J. 1988. Occurrence and pathogenicity of the Streptococcus milleri group. Rev. Infect. Dis. 10:257-285.

2. Limia, A., M.L. Jiménez, T. Alarcón, and M. López-Brea. 1999. Five-year analysis of antimicrobial susceptibility of the Streptococcus milleri group. Eur. J. Clin. Microbiol. Infect. Dis. Off. Publ. Eur. Soc. Clin. Microbiol. 18:440-444.

3. Leclercq, R. 2002. Mechanisms of resistance to macrolides and lincosamides: nature of the resistance elements and their clinical implications. Clin. Infect. Dis. Off. Publ. Infect. Dis. Soc. Am. 34:482-492.

4. Cattoir, V., L. Merabet, P. Legrand, C.-J. Soussy, and R. Leclercq. 2007. Emergence of a Streptococcus pneumoniae isolate resistant to streptogramins by mutation in ribosomal protein L22 during pristinamycin therapy of pneumococcal pneumonia. J. Antimicrob. Chemother. 59:1010-1012.

5. Bingen, E., R. Leclercq, F. Fitoussi, N. Brahimi, B. Malbruny, D. Deforche, and R. Cohen. 2002. Emergence of group A Streptococcus strains with different mechanisms of macrolide resistance. Antimicrob. Agents Chemother. 46:1199-1203.

6. Weisblum, B. 1995. Erythromycin resistance by ribosome modification. Antimicrob. Agents Chemother. 39:577-585.

7. Vester, B., and S. Douthwaite. 2001. Macrolide resistance conferred by base substitutions in 23S rRNA. Antimicrob. Agents Chemother. 45:1-12.

8. Malbruny, B., A. Canu, B. Bozdogan, B. Fantin, V. Zarrouk, S. DutkaMalen, C. Feger, and R. Leclercq. 2002. Resistance to quinupristin-dalfopristin due to mutation of L22 ribosomal protein in Staphylococcus aureus. Antimicrob. Agents 
9. Poyart, C., G. Quesne, S. Coulon, P. Berche, and P. Trieu-Cuot. 1998.

277 Identification of Streptococci to species level by sequencing the gene encoding the manganese-dependent superoxide dismutase. J. Clin. Microbiol. 36:41-47.

10. Newton, C.R., A. Graham, L.E. Heptinstall, S.J. Powell, C. Summers, N. Kalsheker, J.C. Smith, and A.F. Markham. 1989. Analysis of any point mutation in DNA. The amplification refractory mutation system (ARMS). Nucleic Acids Res. $17: 2503-2516$.

11. Ye, S., S. Dhillon, X. Ke, A.R. Collins, and I.N. Day. 2001. An efficient procedure for genotyping single nucleotide polymorphisms. Nucleic Acids Res. 29:E88-88.

12. Wilson, D.N. 2014. Ribosome-targeting antibiotics and mechanisms of bacterial resistance. Nat. Rev. Microbiol. 12:35-48.

13. Roberts, M.C. 2008. Update on macrolide-lincosamide-streptogramin, ketolide, and oxazolidinone resistance genes. FEMS Microbiol. Lett. 282:147-159.

14. Lucier, T.S., K. Heitzman, S.K. Liu, and P.C. Hu. 1995. Transition mutations in the 23S rRNA of erythromycin-resistant isolates of Mycoplasma pneumoniae. Antimicrob. Agents Chemother. 39:2770-2773.

15. Deguchi, T., M. Yasuda, K. Horie, K. Seike, M. Kikuchi, K. Mizutani, T. Tsuchiya, S. Yokoi, M. Nakano, and S. Hoshina. 2015. Drug resistance-associated mutations in Mycoplasma genitalium in female sex workers, Japan. Emerg. Infect. Dis. 21:1062-1064.

16. Tait-Kamradt, A., T. Davies, P.C. Appelbaum, F. Depardieu, P. Courvalin, 
J. Petitpas, L. Wondrack, A. Walker, M.R. Jacobs, and J. Sutcliffe. 2000. Two new mechanisms of macrolide resistance in clinical strains of Streptococcus pneumoniae from Eastern Europe and North America. Antimicrob. Agents Chemother. 44:3395-3401.

17. Depardieu, F., and P. Courvalin. 2001. Mutation in 23S rRNA responsible for resistance to 16-Membered macrolides and streptogramins in Streptococcus pneumoniae. Antimicrob. Agents Chemother. 45:319-323.

18. Mankin, A.S., and R.A. Garrett. 1991. Chloramphenicol resistance mutations in the single 23S rRNA gene of the archaeon Halobacterium halobium. J. Bacteriol. 173:3559-3563.

19. Klappenbach, J.A., P.R. Saxman, J.R. Cole, and T.M. Schmidt. 2001. rrndb: the ribosomal RNA operon copy number database. Nucleic Acids Res. 29:181-184.

20. Matzov, D., Z. Eyal, R.I. Benhamou, M. Shalev-Benami, Y. Halfon, M. Krupkin, E. Zimmerman, H. Rozenberg, A. Bashan, M. Fridman, and A. Yonath. 2017. Structural insights of lincosamides targeting the ribosome of Staphylococcus aureus. Nucleic Acids Res. 45:10284-10292.

\section{Legend of the figure}

Figure 1. A) Partial nucleotide sequence alignment of 23S rRNA ( $r r l$ ) encoding gene. Mutation at position 2062 is framed in bold line (E. coli numbering). B Left) RT-PCR results curves showing CT difference between wild-type (WT) primers vs ARMS 
322 mismatches primers for a MLS susceptible strain of S. constellatus 13422 and the 323 resistant strain UCN96. Green lines corresponding to amplification when using WT 324 primers and red line corresponding to amplification when using mismatches primers. 
A)

235 s. constellatus UCN96

$23 S_{3}$, constellatus 1050

235_s. pnewmonfae

235 E. coll

CGGAAA CECCCATGGAGCTTTACTGCAGTTTGATATTGAGTGTCTGTACCACATGTACA CGGAAA ACCCATGGAGCTTTACTGCAGTTTGATATTGAGTGTCTGTGCCACATGTACA CGGAMA A CCCATGGAGCTITACTGCAGTTTGATATTGAGTGTCTGTACCACATGTACA CGGAAAA

\section{2}

B)

S. constellatus 13422

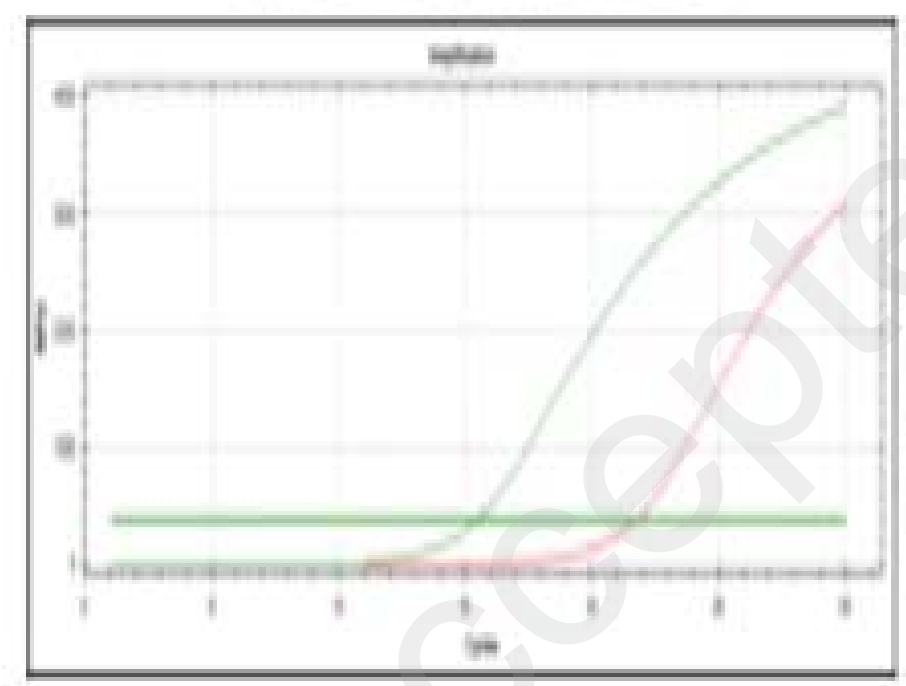

S. constellatus UCN96

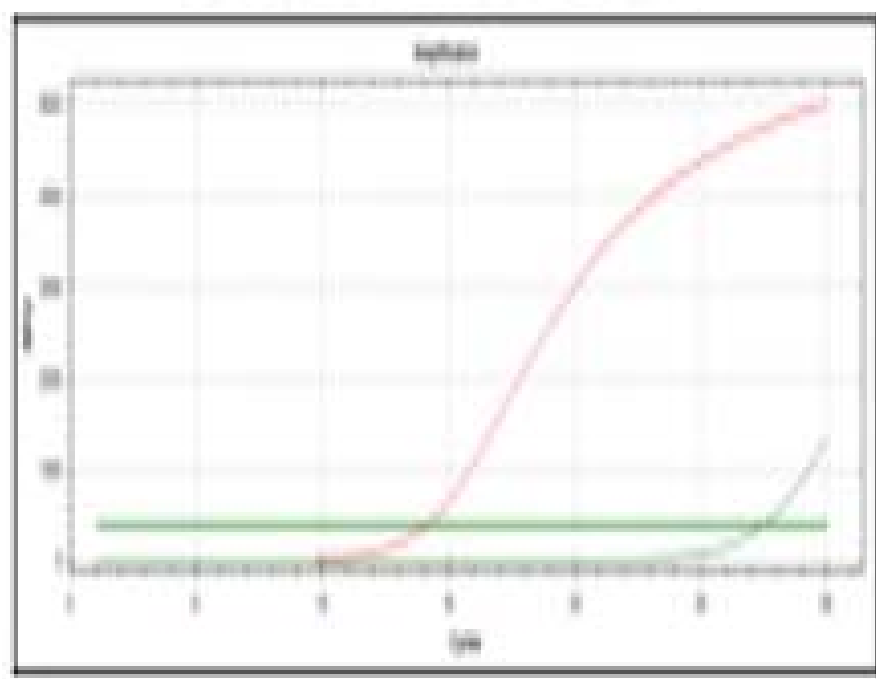


Table 1. Primers used in this study

\begin{tabular}{|c|c|c|c|}
\hline \multirow{2}{*}{$\begin{array}{c}\text { Gene } \\
\text { (ribosomal } \\
\text { protein) }\end{array}$} & \multicolumn{2}{|r|}{ Primer } & \multirow{2}{*}{ Purpose } \\
\hline & Designation & Sequence (5' to $\left.3^{\prime}\right)$ & \\
\hline \multirow{2}{*}{$r p / C(\mathrm{~L} 3)$} & L3-F & CGCCCTTGGTATCTTAATCT & Detection of point mutation in \\
\hline & L3-R & CTTCACCAGCTTCTTTACCAG & L3 protein \\
\hline \multirow{2}{*}{$r p / D(L 4)$} & L4-F & GTGCCTGGTGCTAAGAAATC & Detection of point mutation in \\
\hline & L4-R & TCTTCAAGAAGAGCCATTGA & L4 protein \\
\hline \multirow{2}{*}{$r p / V(L 22)$} & L22-F & CACAAGCTTGGTGAGTTTGC & Detection of point mutation in \\
\hline & L22-R & TACCATTTGGCATCCCAGTC & L22 protein \\
\hline rrl (23S rRNA) & $23 S-F$ & CGAAATTCCTTGTCGGGTAA & Detection of point mutation in \\
\hline domain V & $23 S-R$ & CCGTAGATGATCAACCTAC & domain $\mathrm{V}$ of $23 \mathrm{~S}$ rRNA \\
\hline \multirow{5}{*}{$\begin{array}{c}\text { rrl (23S rRNA) } \\
\text { domain V }\end{array}$} & 23S_ARMS_WT_F1 & CGCGACAGGACGGAAAGA & \\
\hline & 23S_ARMS_WT_F2 & CGCGACAGGACGGAATGA & Allelic determination of point \\
\hline & 23S_ARMS_Mut_F1 & CGCGACAGGACGGAAAGC & mutation in 23S rRNA \\
\hline & 23S_ARMS_Mut_F2 & CGCGACAGGACGGAATGC & (ARMS) \\
\hline & 23S_ARMS_R & ATCCCAACATCGCCTCCATC & \\
\hline
\end{tabular}


Table 2. MICs of MLS antibiotics and Chloramphenicol used in this study

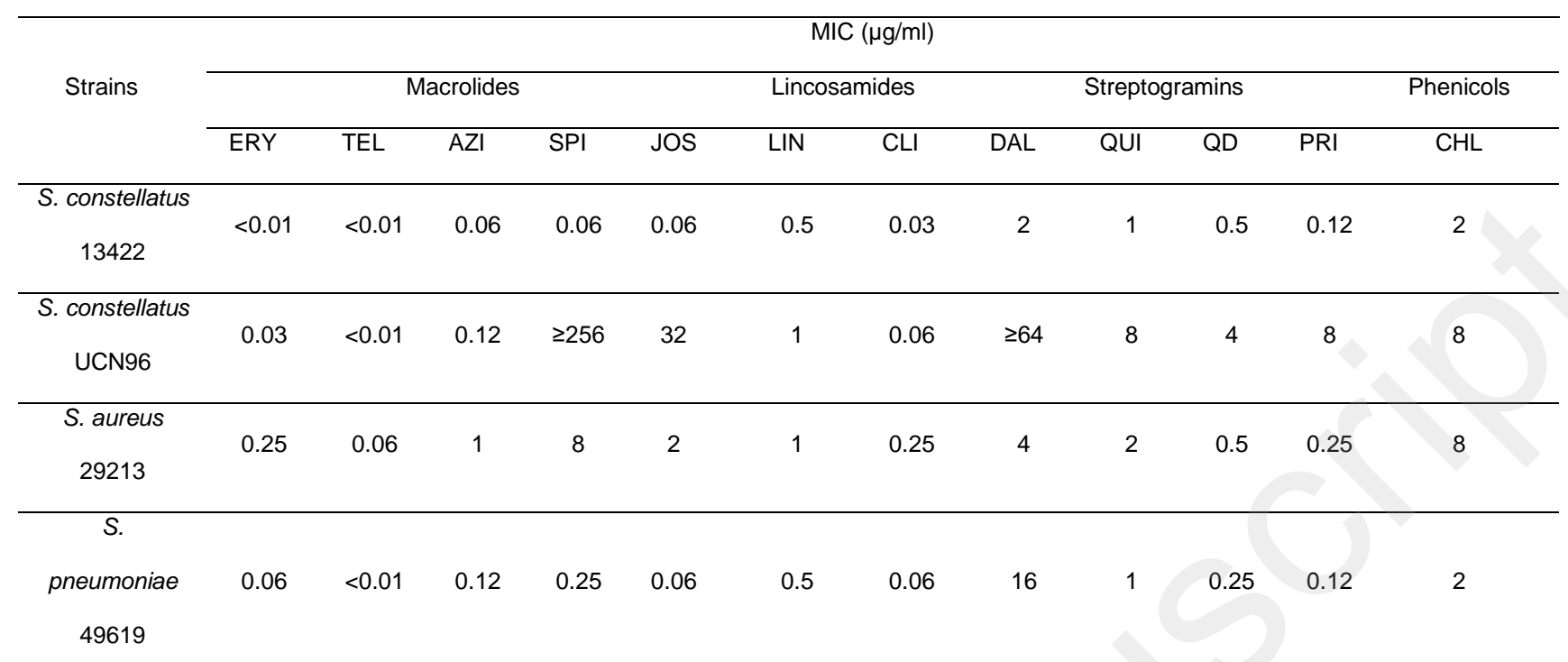

ERY, Erythromycin ; TEL, Telithromycin ; AZI, Azithromycin ; SPI, Spiramycin ; JOS, Josamycin ; LIN, Lincomycin; CLI, Clindamycin;

DAL, Dalfopristin; QUI, Quinupristin; Q-D, Quinupristin-Dalfopristin; PRI, Pristinamycin ; CHL, Chloramphenicol. 